\title{
The spectrum of dengue fever in pregnancy
}

\author{
S. Lalitha ${ }^{1}$, Padmasri R.,", Akhila .M.V ${ }^{3}$, Anjali R $^{4}$ \\ ${ }^{\mathbf{1}}$ Assistant Professor, ${ }^{2}$ Professor and HOD, ${ }^{3,4}$ Senior Resident, Dept. of Obstetrics and Gynaecology, Sapthagiri Institute of \\ Medical Sciences and Research Centre, Bangalore, Karnataka, India
}

*Corresponding Author:

Email: drpadmasuraj@gmail.com

Received: $10^{\text {th }}$ April, 2018

Accepted: $21^{\text {st }}$ May, 2018

\begin{abstract}
Introduction: Dengue is a vector-borne disease transmitted by the mosquito Aedes aegypticus. Dengue infection in pregnancy carries the risk of hemorrhage for both the mother and the newborn.

Materials and Methods: 148 patients with acute febrile illness during Nov 2015 to Nov 2017 were analysed. Data regarding course of illness, lab reports which included complete blood count, urine routine and culture, NS1 Antigen, WIDAL and Peripheral smear for malarial parasite, treatment received, maternal and perinatal outcome were analysed from records.

Results: NS1 Antigen was detected in $40 \%$ of all cases with fever. Amongst the remaining, $15 \%$ had viral fever, $14 \%$ had urinary tract infection, $10 \%$ had enteric fever, $8 \%$ had lower respiratory tract infection, $2 \%$ had malaria and $9 \%$ had fever of unknown origin. Thrombocytopenia was present in $40 \%$ of dengue positive patients and $30 \%$ of patients required transfusion. 2 patients diagnosed close to term had postpartum hemorrhage treated with platelets and FFPs. There were two neonatal deaths.

Conclusion: Though most cases required only conservative management one has to keep in mind the correlation of period of gestation to the outcome and complications.
\end{abstract}

Keywords: Dengue, Pregnancy, Adverse effects.

\section{Introduction}

Dengue is an increasing problem in the tropical world. It is one of the most important arboviral infections effecting mankind. Every year there are between 50 and 100 million cases of dengue fever and 500,000 cases of dengue hemorrhagic fever worldwide.

Dengue is a self-limiting febrile illness. But, lack of early recognition and symptomatic treatment causes complications like Dengue Hemorrhagic Fever (DHF) and Dengue Shock Syndrome (DSS). Pregnancy is a very vulnerable period and is associated with several physiological changes. ${ }^{1}$ Dengue fever during pregnancy causes maternal complications like hemorrhage and abortion and fetal complications like pre-term babies, intra-uterine death (IUD), and low birth-weight babies. ${ }^{2}$

Recent frequent epidemics have made it a serious public health problem. The disease is traditionally caused by bite of Aedes aegypti mosquito and the illness can vary from merely asymptomatic to one with life-threatening dengue hemorrhagic fever. There are four serotypes of dengue and presumably infection with one serotype does not lead to immunity against the other. Rather the chances of developing a severe form of the disease are more if prior sensitization has occurred with a different serotype. Dengue is defined by the World Health Organization (WHO) as an acute febrile illness associated with two or more of the following signs or symptoms: intense headache, retroorbital pain, myalgia, arthralgia, skin rash, leucopenia and hemorrhagic manifestations. ${ }^{3}$ Given the incidence during dengue epidemics, a significant number of pregnant women also get infected with dengue.
However, there are not many studies on the obstetrical consequences of dengue during pregnancy.

In most of the cases of dengue fever in pregnancy, no serious harm has been noted. Treatment includes proper hydration, antipyretics and careful monitoring. Literature search reveals an increased incidence of preterm deliveries, low birth weight, pre-eclampsia and caesarean sections. Vertical transmission was also noted $^{4}$. During the dengue epidemic in our region, we came across many pregnant women with dengue fever and found certain features not mentioned in other studies. With this background, we studied the pregnant women with dengue fever who were admitted in the obstetric ward and determined the adverse effects of dengue fever on pregnancy and the fetal outcome.

\section{Materials and Methods}

Study Design: Cross-Sectional study using secondary data.

Study Population: The records of all pregnant mothers who came for antenatal check-up and had history of fever, between November 2015 - November 2017, (24 months) were retrieved and data regarding lab investigations like Dengue NS1 antigen, WIDAL, peripheral smear for malarial parasite, complete blood counts, urine routine and culture were noted.

Dengue was diagnosed according to WHO definition: an acute febrile illness with 2 or more clinical manifestation like headache, retro-orbital pain, myalgia, arthralgia, rash, hemorrhagic manifestation, or leucopenia and positive serology or occurrence at the time of dengue outbreak. We used NS1 antigen and / or 
IgM serology. Clinical grading was done according to WHO classification and case definition.

Analysis was done with respect to age of the patient, gestational age, complications at presentation, laboratory diagnosis, platelet counts and treatment offered and pregnancy outcome were noted.

\section{Results}

A total of 148 pregnant women with acute febrile illness were identified out of which 60 cases were found to have been infected with dengue virus.

Among the patients with dengue fever, the most common mode of presentation was fever in 50 pregnant women $(83.3 \%)$. fever with myalgia in $10 \%$ (6 cases) and abdominal pain in $6.7 \%$ (4 cases).

Of the 60 women with dengue fever in pregnancy, primary dengue was seen in 22 patients $(43.3 \%)$, where NS1 antigen was found to be positive and secondary dengue infection were seen in the remaining 38 patients (63.3\%). 4 cases $(5.83 \%)$ had co-incidental infection with typhoid.

The mean age of women with dengue infection was 28 years, the youngest being 19 years and the oldest being 34 years. Majority of patients $(56.7 \%)$ were in the age group of 25-30 years. Regarding gestational age, 24 patients $(40 \%)$ were in the gestational age between 28 36 weeks, 16 patients $(26.7 \%)$ were between 13-27 weeks of gestational age and 20 patients $(33.3 \%)$ were beyond 36 weeks. Out of 20 patients who presented in the late trimester, 4 patients had evidence of hypertensive disorder of pregnancy and 2 patients developed post-partum haemorrhage which was managed conservatively. None of the patients required ICU admission.

A hematocrit level increase greater than $20 \%$ is a sign of hemoconcentration and precedes shock. Fig. 1 shows the PCV values of 60 dengue patients. In this study, $10 \%$ of patients had hematocrit value of $40 \%$ and $23.3 \%$ had hematocrit value of $42 \%$. Taking into account the normal hematocrit values during pregnancy as $31-40 \%$ in different trimesters, we can say $23.3 \%$ of patients had hemoconcentration. Those who had high PCV values had low platelet counts and this accounted for the development of dengue hemorrhagic fever and post-partum hemorrhage and the need for blood transfusion in such cases.

Table 1 shows the values of liver enzymes. All patients had elevation in liver enzymes with AST levels being higher than ALT levels, though the number of patients who had PPH was only 4 . This could be due to early intervention with platelet and blood component transfusion wherever required.

Out of 60 dengue positive pregnant women, 22 patients required platelet transfusion as seen in Table 2. Among them, 4 patients were transfused with single donor platelets as counts were below 40,000 and these required immediate termination of pregnancy due to obstetric reasons.
Table 3, 4 and 5 shows the significant association between the gestational age at presentation, severity of dengue fever and the need for platelet transfusion with the mode of delivery.

Out of 60 patients, 24 patients (40\%) had full term vaginal delivery, 16 patients underwent caesarean section due to obstetric indications, 8 patients $(33.3 \%)$ had pre-term vaginal delivery and 8 patients were lost for follow up. Among those patients who had pre-term deliveries, hyperthermia due to dengue fever could be the precipitating factor. The women, who presented with dengue early in pregnancy between 13 to 27 weeks, improved and were discharged. They did not come for further follow-up at our hospital or deliver here so the outcome of dengue in early pregnancy could not be estimated. Those who developed dengue in $3^{\text {rd }}$ trimester, majority of them delivered vaginally. Among them, 4 patients had post partum hemorrhage. There were 2 neonatal deaths, the cause being pre-term delivery and respiratory distress syndrome. Those patients with dengue at term had a $20 \%$ incidence of emergency caesarean section, the indication being non reassuring fetal heart rates. Meconium was found 8 of these patients. The most common indication for elective caesarean section was previous caesarean section. Neonatal outcome in these women was relatively good.

Table 4 describes the association between severity of dengue infection and its outcome. In this study, $33.33 \%$ (i.e. 20 patients) had dengue fever. In that, majority i.e. 16 patients delivered vaginally. 4 patients had associated pregnancy induced hypertension, all of them had pre-term vaginal delivery, in that two patients progressed spontaneously and two patients were induced. Among 32 patients who had dengue hemorrhagic fever, 12 patients landed up with emergency caesarean section due to obstetric indication. 4 patients had post-partum hemorrhage which was managed conservatively with blood transfusion.

Table 5 describes the need for platelet transfusion among the dengue cases. Out of 60 patients with dengue fever, 29 patients required blood component transfusion. Out of these, 12 patients had severe thrombocytopenia i.e. platelet count of $<50,000 /$ cumm, and needed 2-3 pints of platelet transfusion. 1 patient who had platelet count of $28,000 /$ cumm required 5 pints of single donor platelets (SDP). 8 patients required Fresh Frozen Plasma transfusion. The neonatal outcome was relatively good. Even in patients with severe thrombocytopenia, platelet transfusion was done only in those patients who had gone into labour or who had to undergo emergency or elective caesarean section. 
Table 1: Frequency of elevation of liver enzyme values among the patients

\begin{tabular}{|l|c|c|}
\hline AST/ALT & Number of cases & Percentage (\%) \\
\hline $108 / 60$ & 2 & 3.3 \\
\hline $154 / 100$ & 2 & 3.3 \\
\hline $154 / 116$ & 2 & 3.3 \\
\hline $155 / 64$ & 2 & 3.3 \\
\hline $156 / 40$ & 2 & 3.3 \\
\hline $156 / 60$ & 10 & 16.7 \\
\hline $156 / 64$ & 2 & 3.3 \\
\hline $156 / 70$ & 2 & 3.3 \\
\hline $157 / 40$ & 2 & 3.3 \\
\hline $158 / 110$ & 2 & 3.3 \\
\hline $158 / 40$ & 6 & 10 \\
\hline $158 / 58$ & 6 & 10 \\
\hline $158 / 60$ & 6 & 10 \\
\hline $158 / 70$ & 6 & 10 \\
\hline $160 / 40$ & 4 & 6.7 \\
\hline $160 / 60$ & 2 & 3.3 \\
\hline $172 / 40$ & 2 & 3.3 \\
\hline Total & 60 & 100 \\
\hline
\end{tabular}

Table 2 : Platelet counts and need for platelet transfusion

\begin{tabular}{|l|c|c|}
\hline $\begin{array}{c}\text { Platelet count on } \\
\text { admission (cells/mm3) }\end{array}$ & $\begin{array}{c}\text { No. of pregnant } \\
\text { women }\end{array}$ & $\begin{array}{c}\text { No. of patients who } \\
\text { received transfusion }\end{array}$ \\
\hline $30,000-50,000$ & 12 & 12 \\
\hline $50,000-1$ lakh & 12 & 2 \\
\hline$>1$ lakh & 36 & 8 \\
\hline Total & 60 & 22 \\
\hline
\end{tabular}

Table 3 : Association between gestational age and mode of delivery

\begin{tabular}{|c|c|c|c|c|c|c|c|c|}
\hline & \multicolumn{6}{|l|}{ MOD } & \multirow[b]{2}{*}{ Total } \\
\hline & & $\begin{array}{c}\text { Elective } \\
\text { LSCS }\end{array}$ & $\begin{array}{c}\text { Emergency } \\
\text { LSCS }\end{array}$ & FTVD & $\begin{array}{c}\text { FTVD } \\
\text { PPH }\end{array}$ & $\begin{array}{l}\text { Lost for } \\
\text { follow }\end{array}$ & PTVD & \\
\hline \multirow{6}{*}{$\begin{array}{l}\text { Gestational } \\
\text { age }\end{array}$} & \multirow[t]{2}{*}{ 13-27 weeks } & 0 & 0 & 0 & 0 & 4 & 0 & 4 \\
\hline & & $0.0 \%$ & $0.0 \%$ & $0.0 \%$ & $0.0 \%$ & $6.67 \%$ & $0.0 \%$ & $6.67 \%$ \\
\hline & \multirow[t]{2}{*}{ 28-36 weeks } & 0 & 0 & 8 & 0 & 4 & 8 & 20 \\
\hline & & $0.0 \%$ & $0.0 \%$ & $13.33 \%$ & $0.0 \%$ & $6.67 \%$ & $13.33 \%$ & $33.33 \%$ \\
\hline & \multirow[t]{2}{*}{$>36$ weeks } & 4 & 12 & 16 & 4 & 0 & 0 & 36 \\
\hline & & $6.67 \%$ & $20 \%$ & $26.67 \%$ & $6.67 \%$ & $0.0 \%$ & $0.0 \%$ & $60 \%$ \\
\hline \multirow{2}{*}{\multicolumn{2}{|c|}{ Total }} & 4 & 12 & 24 & 4 & 8 & 8 & 60 \\
\hline & & $6.67 \%$ & $20 \%$ & $40 \%$ & $6.67 \%$ & $13.33 \%$ & $13.33 \%$ & $100.0 \%$ \\
\hline
\end{tabular}

P-value -0.02

Table 4 : Association between severity of dengue fever and mode of delivery

\begin{tabular}{|c|c|c|c|c|c|c|c|c|}
\hline & \multicolumn{6}{|l|}{ MOD } & \multirow[b]{2}{*}{ Total } \\
\hline & & $\begin{array}{c}\text { Elective } \\
\text { LSCS }\end{array}$ & $\begin{array}{l}\text { Emergency } \\
\text { LSCS }\end{array}$ & FTVD & $\begin{array}{l}\text { FTVD } \\
\text { PPH }\end{array}$ & $\begin{array}{l}\text { Lost for } \\
\text { follow up }\end{array}$ & PTVD & \\
\hline \multirow[t]{8}{*}{ Severity } & \multirow[t]{2}{*}{ Dengue fever } & 0 & 0 & 16 & 0 & 4 & 0 & 20 \\
\hline & & $0.0 \%$ & $0.0 \%$ & $26.67 \%$ & $0.0 \%$ & $6.67 \%$ & $0.0 \%$ & $33.33 \%$ \\
\hline & \multirow{2}{*}{$\begin{array}{c}\text { Dengue fever + } \\
\text { PIH }\end{array}$} & 0 & 0 & 0 & 0 & 0 & 4 & 4 \\
\hline & & $0.0 \%$ & $0.0 \%$ & $0.0 \%$ & $0.0 \%$ & $0.0 \%$ & $6.67 \%$ & $6.67 \%$ \\
\hline & \multirow[t]{2}{*}{ DHF } & 4 & 12 & 8 & 0 & 4 & 4 & 32 \\
\hline & & $6.67 \%$ & $20 \%$ & $13.33 \%$ & $0.0 \%$ & $6.67 \%$ & $6.67 \%$ & $53.33 \%$ \\
\hline & \multirow[t]{2}{*}{$\mathrm{PIH}+\mathrm{PPH}$} & 0 & 0 & 0 & 4 & 0 & 0 & 4 \\
\hline & & $0.0 \%$ & $0.0 \%$ & $0.0 \%$ & $6.67 \%$ & $0.0 \%$ & $0.0 \%$ & $6.67 \%$ \\
\hline
\end{tabular}




\begin{tabular}{|l|c|c|c|c|c|c|c|}
\hline \multirow{2}{*}{ Total } & 4 & 12 & 24 & 4 & 8 & 8 & 60 \\
\cline { 2 - 8 } & $6.67 \%$ & $20 \%$ & $40 \%$ & $6.67 \%$ & $13.33 \%$ & $13.33 \%$ & $100.0 \%$ \\
\hline
\end{tabular}

P-value -0.001

DF- Dengue Fever, DHF- Dengue Hemorrhagic Fever, PPH - Post-Partum Hemorrhage, PIH - Pregnancy Induced Hypertension.

Table 5 : Association between platelet transfusion and mode of delivery

\begin{tabular}{|c|c|c|c|c|c|c|c|c|}
\hline & \multicolumn{6}{|l|}{ MOD } & \multirow[b]{2}{*}{ Total } \\
\hline & & $\begin{array}{c}\text { Elective } \\
\text { LSCS }\end{array}$ & $\begin{array}{c}\text { Emergenc } \\
\text { y LSCS }\end{array}$ & FTVD & $\begin{array}{c}\text { FTVD } \\
\text { PPH }\end{array}$ & $\begin{array}{c}\text { Lost for } \\
\text { follow }\end{array}$ & PTVD & \\
\hline \multirow[t]{12}{*}{$\mathrm{Pl}$ transfusion } & \multirow[t]{2}{*}{2 Platelets } & 0 & 0 & 4 & 0 & 0 & 4 & 8 \\
\hline & & $0.0 \%$ & $0.0 \%$ & $6.67 \%$ & $0.0 \%$ & $0.0 \%$ & $6.67 \%$ & $13.33 \%$ \\
\hline & \multirow{2}{*}{$\begin{array}{c}2 \text { PRBC +1 } \\
\text { FFP }\end{array}$} & 0 & 0 & 0 & 4 & 0 & 0 & 4 \\
\hline & & $0.0 \%$ & $0.0 \%$ & $0.0 \%$ & $6.67 \%$ & $0.0 \%$ & $0.0 \%$ & $6.67 \%$ \\
\hline & \multirow[t]{2}{*}{3 platelets } & 0 & 8 & 4 & 0 & 0 & 0 & 12 \\
\hline & & $0.0 \%$ & $13.33 \%$ & $6.67 \%$ & $0.0 \%$ & $0.0 \%$ & $0.0 \%$ & $20 \%$ \\
\hline & \multirow{2}{*}{$\begin{array}{c}3 \mathrm{PRBC}+3 \\
\text { FFP }\end{array}$} & 0 & 0 & 4 & 0 & 0 & 0 & 4 \\
\hline & & $0.0 \%$ & $0.0 \%$ & $6.67 \%$ & $0.0 \%$ & $0.0 \%$ & $0.0 \%$ & $6.67 \%$ \\
\hline & \multirow[t]{2}{*}{5 SDP } & 0 & 0 & 0 & 0 & 0 & 1 & 1 \\
\hline & & $0.0 \%$ & $0.0 \%$ & $0.0 \%$ & $0.0 \%$ & $0.0 \%$ & $1.67 \%$ & $1.67 \%$ \\
\hline & \multirow[t]{2}{*}{ Nil } & 4 & 4 & 12 & 0 & 8 & 3 & 31 \\
\hline & & $6.67 \%$ & $6.67 \%$ & $20 \%$ & $0.0 \%$ & $13.33 \%$ & $5 \%$ & $51.67 \%$ \\
\hline \multirow{2}{*}{\multicolumn{2}{|c|}{ Total }} & 4 & 12 & 24 & 4 & 8 & 8 & 60 \\
\hline & & $6.67 \%$ & $20 \%$ & $40 \%$ & $6.67 \%$ & $13.33 \%$ & $13.33 \%$ & $100.0 \%$ \\
\hline
\end{tabular}

P-value $-0.02 \mathrm{~s}$

PRBC - Packed red blood cell, FFP - Fresh Frozen Plasma, SDP - Single donor Platelets

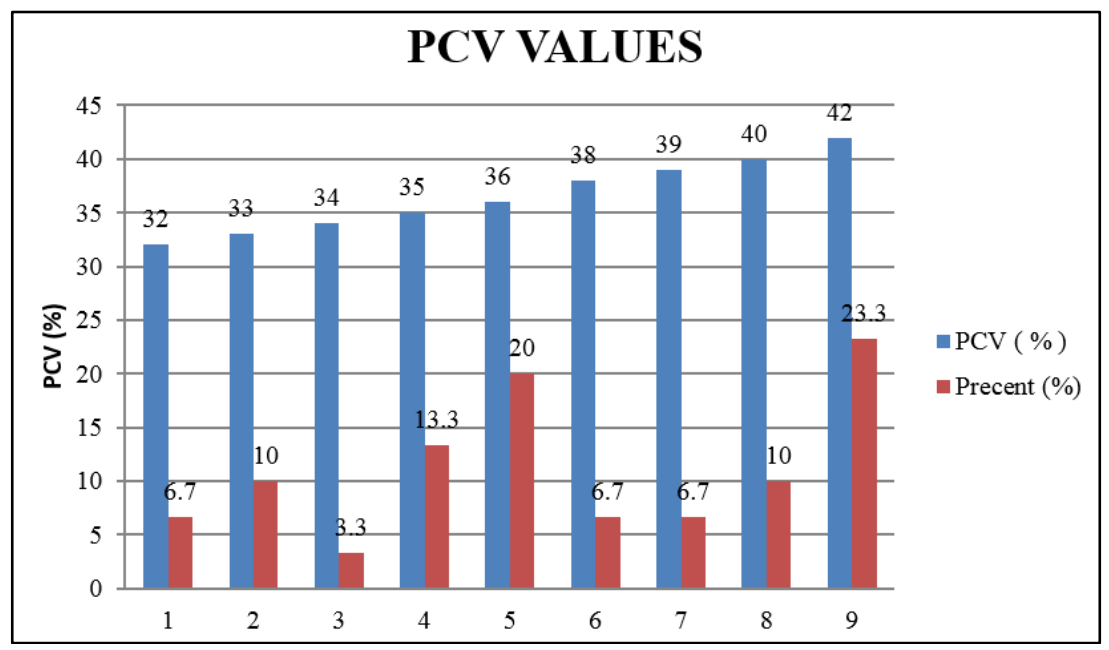

Fig. 1 : PCV values of women with dengue

\section{Discussion}

Fever with thrombocytopenia during pregnancy causes panic among the practicing obstetricians. It is important to consider dengue as a possible differential diagnosis of acute febrile illness in endemic region. ${ }^{4}$ In general, the most common symptoms include fever, myalgia and arthralgia. Abdominal pain and vomiting were seen in a few cases. ${ }^{4}$

Severe symptoms like hepatic cytolysis or severe thrombocytopenia were reported without dengue hemorrhagic fever (DHF). However, the physiological changes that occur during pregnancy such as haemodilution or ability to coagulate may mask thrombocytopenia, leucopoenia, or a hematocrit increase. In addition, hepatic and haematological problems can also be observed with other obstetric complications such as morning sickness or HELLP syndrome, which may result in an underestimation of dengue.

The mean age of women was $28+/-6$ yrs, the youngest being 19 yrs and the oldest being $34 \mathrm{yrs}$ in our study. The mean gestational age at presentation was $28.31+/-8.48$ wks, with lowest being 18 wks and the highest being 40wks. The gestational age at presentation of dengue fever appeared to be significant. 
The trimester in which dengue infection occurs apparently affects the rate of adverse outcome, so that mother infected in first trimester has a higher risk of fetal death. However, since women in early pregnancy may not be hospitalized, the frequency of vertical transmission remains difficult to estimate. Zavatonni reports a case of an acute dengue viral infection in the first weeks of pregnancy to an unfavorable outcome. ${ }^{5}$ It should be borne in mind, however, that one in five of all recognized pregnancies end in miscarriage ${ }^{6}$ and maternal hyperthermia is recognized as an independent risk factor for miscarriage. However, when the infection occurs in the third trimester, the risks of low birth weight, premature labor and vertical transmission increase. Kanakalatha DH et al. ${ }^{7}$ had done a prospective study in 73 cases of maternal dengue, where 15 patients presented before 20 weeks. Among them, 4 had spontaneous abortion, 4 patients had platelet counts less than $50,000 / \mathrm{mm} 3$ of which 2 patients had developed preeclampsia later in pregnancy and required termination of pregnancy before 37 weeks. All the other patients had otherwise uncomplicated pregnancy and term delivery. 22 patients presented between 20 to 34 weeks, 7 patient had preterm deliveries, 1 patient had DHF, 1 had DSS and all others had term deliveries. In their study, $30.1 \%$ were infected after 37 weeks- among them $8.8 \%$ patients had PPH, $7.3 \%$ had postpartum fever, $7.3 \%$ required ICU admission and $2.9 \%$ had respiratory distress in the postpartum period.

In our study, the number of women who presented beyond $31 \mathrm{wks}$ was 44 out of 60.3 of them had evidence of Pregnancy Induced Hypertension. In our study, 1 patient was referred at 33 wks with fever. She had a platelet count of 28,000 cells/cumm and she received 5 units of single donor platelets.

In our study, 4 patients diagnosed close to term had post-partum hemorrhage and were treated with platelets and fresh frozen plasma (FFP). In a retrospective study by Carlos et $\mathrm{al}^{13}$ on 82 pregnant dengue patients, four cases $(30.8 \%)$ had obstetric hemorrhage.

In our study, the striking feature was severe thrombocytopenia (platelet count of $<50,000$ cells/cumm) which was seen in 12 out of 60 patients. The fall in the platelet count was rapid initially. Platelet transfusion was done only if the mother went into labour or had any bleeding tendencies or if she was posted for caesarean section. Similar results were seen with the study by Chitra et $\mathrm{al}^{4}$ where the pregnant women with dengue were treated conservatively, with platelet transfusion being given only during labour or in case of bleeding tendencies.

No cases of dengue shock syndrome were noted in our study.

Low birth weight and pre-term deliveries have been noted in various studies. A clinical study done by Swati Sharma et $\mathrm{al}^{8}$ had pre-term deliveries in $12 \%$ of the cases. The average birth weight noted in our study was $2.44 \mathrm{kgs}$. Gehlot $\mathrm{H}$ et $\mathrm{al}^{9}$ reported an average birth weight of $<2.5 \mathrm{~kg}$ in $16 \%$ cases. $8 \%$ of babies required NICU admission and $4 \%$ had IUFD. Symptomatic dengue infection during pregnancy may increase the risk of PTB and LBW for infants. There were 2 neonatal deaths in our study, the reason being pre-term delivery and respiratory distress syndrome.

The average incubation period of dengue fever is estimated to be about 7 days. Dengue serology was not performed on normal babies and hence no comment could be made on vertical transmission.

Though rare, dengue viral infection can result in mortality. Although morbidity like PIH, PPH and prolonged fever were noted in our study, there was no maternal death. Helena et $\mathrm{al}^{10}$ reported a maternal mortality of $1 \%$ in the exposed cohort and none in the unexposed cohort $(\mathrm{P}=0.040)$. Of the two maternal deaths, one was directly related to dengue virus infection. According to a study in Rio de Janeiro by Machado et al, ${ }^{11}$ pregnant women were 3.4 times more likely to develop severe dengue, and mortality was higher in pregnant than in non-pregnant women, Therefore, any pregnant woman with an acute febrile illness, especially in endemic areas, should be investigated for dengue virus infection, since the increased probability of evolution to the severe from during pregnancy requires closer surveillance of pregnant women infected with the virus. ${ }^{12}$

\section{Conclusion}

Dengue infection has emerged to be a major health problem in tropical countries. Though most of the dengue cases require only conservative management, one has to keep in mind the correlation of period of gestation to outcome and complications - early or late pregnancy had a poor prognosis. A high index of clinical suspicion is essential in any pregnant female with fever during epidemics. Travel during pregnancy to dengue endemic areas poses a risk to both mother and fetus, hence requiring close monitoring for potential maternal and fetal complication. Further studies are mandatory as evidence- based data in the management of dengue specific for pregnancy are sparse.

\section{References}

1. Pavanaganga A, Sailakshmi MPA, Rekha BR, Nagarathnamma R. Dengue Fever during pregnancy: maternal and fetal complications. J South Asian Feder Obst Gynae. 2017;9(2):88-91.

2. Agarwal P, Garg R, Srivastava S, Verma V, Rani R. Pregnancy outcome in women with dengue infection in Northern India. Indian J Clin Practice. 2014;24(11);1053-1056.

3. XueruYIN, Xiaozhu ZHONG \& Shilei PAN. Vertical Transmission of Dengue Infection: The First Putative Case Reported In China. Rev Inst Med Trop. Sao Paulo 2016;58:90 http://dx.doi.org/10.1590/S16789946201658090. 
4. Chitra TV, Panicker S. Maternal and fetal outcome of dengue fever in pregnancy. J vector Borne Dis. 2011;48(4):210-213.

5. Zavattoni, M., Rovida, F., Campanini, G., Percivalle, E., Sarasini, A., Cristini, G., Baldanti, F. (2016). Miscarriage following dengue virus 3 infection in the first six weeks of pregnancy of a dengue virus-naive traveller returning from Bali to Italy, April 2016. Eurosurveillance. 21(31), 30308 .

http://doi.org/10.2807/15607917.ES.2016.21.31.3008.

6. Giakoumelou S, Wheelhouse N, Cuschieri K, Entrican G, Howie SE, Horne AW. The role of infection in miscarriage. Hum Reprod Update. 2016;22(1):116-33.

7. Divyasree Harikumar, Kanakalatha, Sreekumary Radha and Bindu Nambisan. Maternal and fetal outcome of dengue fever during pregnancy. Int J Reprod Contraception Obstet Gynecol. 2016;5(11):3959-3964.

8. Swati Sharma, Sandhya Jain, and Shalini Rajaram. Spectrum of Maternofetal Outcomes during Dengue Infection in Pregnancy: An Insight. Infectious Diseases in Obstetrics and Gynecology, vol. 2016, Article ID 5046091, 4 pages, 2016. doi:10.1155/2016/

9. Hanslata Gehlot, Om Prakash Yadav, Seema Sharma, Girdhar Gopal Nagar, Ayua Yadav and Prabhu Prakash Gupta. A study of dengue fever in pregnancy and its maternal and fetal prognosis: International Journal of reproduction, Contraception, Obstetrics and Gynecology. 2017;6(8);3414-3417.
10. Feitoza, Helena Albuquerque Catao, Koifman, Sergio, Koifman, Rosalina Jorge \& Saraceni, Valeria (2017). Dengue infection during pregnancy and adverse maternal, fetal, and infant health outcomes in Rio Branco, Acre State, Brazil, 2007-2012, Cadernos dr Saude Publica, 33(5), e00178915. Epub June 12,

2017..https://dx.doi.org/10.1590/0102-311x00178915.

11. Machado CR, Machado ES, Ronloff RD, Azevedo M. Campus DP, Oliveria RB, et al. Is pregnancy associated with severe dengue? A review of data from the Rio de Janeiro Surveillance Information System, PLoSNegl Trop Dis. 2013;7:e2217.

12. Sellahewa KH, Marasinghe DKU, Najimudeen $M$. Dengue in pregnancy - management perspectives. Journal of Medical Sciences \& Medicine. 2013;1:12-8.

13. Carlos Machain-Williams, Eric Raga, Carlos M. BaakBaak, Sungmin Kiem, Bradley J. Blitvich, and Celso Ramos. Maternal, Fetal, and Neonatal Outcomes in Pregnant Dengue Patients in Mexico. BioMed Research International, vol. 2018, Article ID 9643083, 8 pages, 2018. doi: $10.1155 / 2018 / 9643083$.

How to cite this article: Lalitha S., Padmasri R., Akhila M.V., Anjali R. The spectrum of dengue fever in pregnancy. Ind J Obstet Gynecol Res. 2018;5(3):399-404. 